\title{
Efeitos da Utilização de Diferentes Covariáveis na Avaliação do Ganho de Peso Médio Diário em Suínos ${ }^{1}$
}

\author{
Fabiano Veraldo da Costa Pita2 ${ }^{2}$ Lucia Galvão de Albuquerque ${ }^{3,4}$
}

\begin{abstract}
RESUMO - O objetivo do presente trabalho foi comparar e avaliar possíveis implicações da utilização, como covariáveis, das características idade ao início do teste (IDIN), idade ao final do teste (IDFI), peso ao início do teste (PEIN) e peso ao final do teste (PEFI) na análise do ganho de peso médio diário (GMD) de suínos Landrace (LD), Large White (LW) e Pietrain (PT). Análises unicaracterísticas e bicaracterísticas foram desenvolvidas pelo método de Máxima Verossimilhança Restrita, utilizando-se um modelo que incluiu os efeitos fixo de grupo contemporâneo e aleatórios genéticos aditivo direto e materno (este último apenas para LD) e de leitegada. As correlações genéticas entre PEIN e GMD e entre PEFI e GMD foram de, respectivamente, 0,46 e 0,96 para LD, 0,40 e 0,96 para LW e de 0,51 e 0,97 para PT. As estimativas de herdabilidade para GMD foram semelhantes para as três raças e próximas de 0,28 e 0,10 , respectivamente, quando incluídas no modelo as covariáveis IDIN ou PEFI. A utilização da IDIN ou IDFI como covariável permitiria a comparação dos animais quanto ao GMD em igualdade de condições (mesma idade fisiológica), sem afetar sua análise genética, enquanto a utilização do PEIN ou PEFI (principalmente este último) como covariável provocaria grande redução na variabilidade genética do GMD existente nas populações estudadas.
\end{abstract}

Palavras-chave: idade ao final do teste, idade ao início do teste, peso ao final do teste, peso ao início do teste

\section{Effects of Different Covariates Utilization for Average Daily Gain Evaluation in Swine}

ABSTRACT - The aim of this study was to compare and evaluate the use of on-test age (ONA), off-test age (OFA), on-test weight (ONW), and off-test weight (OFW) as covariates for analysing average daily gain (ADG) in Landrace (LD), Large White (LW) and Pietrain (PT) swine breeds. One- and two-traits analyses were carried out using Restrict Maximum Likelihood method, with a statistical model that included the fixed effect of contemporary group, the random genetic effects of animal and maternal (the latter just for LD breed), and the random effect of litter. Genetic correlations between ONW and ADG and, OFW and ADG were, respectively, 0.46 and 0.96 for LD, 0.40 and 0.96 for $\mathrm{LW}$, and 0.51 and 0.97 for PT. The heritability estimates for ADG were similar for the three breeds and around 0.28 and 0.10 , respectively, when ONA and OFW were included as covariates in the model. The use of ONA or OFA would permit the comparison of animals ADG at the same physiologic age, without affecting genetic analyses. Using ONW or OFW (mainly the latter) would cause large decrease on genetic variability of ADG in the populations studied.

Key Words: off-test age, off-test weight, on-test age, on-test weight

\section{Introdução}

As diferenças de desempenho observadas entre os indivíduos são resultado da influência de fatores genéticos e ambientais.

O componente genético é estritamente uma função dos genes que o indivíduo possui. Embora a seleção de animais em um programa de melhoramento genético seja realizada pelo componente genético, ênfase deve ser dada ao componente ambiental, pois os efeitos ambientais, apesar de não serem transmitidos através das gerações, devem ser considerados para a determinação precisa do valor genético do indivíduo.
A característica de desempenho ganho de peso médio diário (GMD) é influenciada por vários efeitos genéticos e de meio, principalmente raça e sexo, entre outros (COSTA et al., 1986; SILVA et al., 1992b; GUERRA et al., 1992).

Alguns destes efeitos são predizíveis e podem ter influência sobre o desempenho dos indivíduos considerada, por meio da sua inclusão nos modelos de análise como efeitos fixos ou fazendo-se uso de fatores de correção.

A consideração de alguns efeitos ambientais predizíveis como covariáveis tem por finalidade permitir que os animais sejam comparados e avaliados

\footnotetext{
1 Parte da dissertação de Mestrado do primeiro autor (Curso de pós-graduação em Zootecnia, área de concentração em Genética e Melhoramento Animal, Fac. de Ciências Agrárias e Veterinárias, UNESP/Campus de Jaboticabal), financiada pela FAPESP.

2 Zootecnista. Endereço atual:Universidade Federal de Viçosa, curso de pós-graduação em Genética e Melhoramento. E.mail:fvcpita@bol.com.br

3 Professora assistente doutora. FCAV - UNESP/Jaboticabal. Depto de Zootecnia, setor de Melhoramento Animal. E.mail: Igalb@fcav.unesp.br 4 Pesquisadora do CNPq.
} 
em igualdade de condições para estes efeitos, pois influem diretamente sobre o desempenho no período de teste dos indivíduos.

No caso particular da análise do ganho de peso médio diário de suínos, durante o teste de granja, não existe, na literatura, concordância com relação à qual característica deve ser utilizada como covariável. Alguns autores utilizaram como covariáveis o peso ao início e ao final do teste (LO et al., 1992; BIDANEL e DUCOS, 1996), enquanto outros utilizaram apenas o peso ao início do teste (DUCOS et al., 1993; BRAZÃO, 1997) ou ao final do teste (HOFER et al., 1992). Também a idade ao início do teste foi utilizada como covariável para estudo do GMD, por COSTA et al. (1986) e SILVA et al. (1992b), e a idade ao final do teste, por JEFFRIES e PETERSON (1982). ROSO (1997a, b) utilizou a idade ao início e o peso ao final do teste, enquanto GU et al. (1989) utilizaram a idade e o peso ao início do teste conjuntamente com o tempo de duração do mesmo. Por outro lado, diversos autores não incluem covariável alguma no modelo de análise do GMD (MERKS, 1987; CAMERON, 1990; BRANDT e TÄUBERT, 1998).

O objetivo do presente trabalho foi comparar e avaliar possíveis implicações da utilização, como covariáveis, das características idade ao início do teste (IDIN), idade ao final do teste (IDFI), peso ao início do teste (PEIN) e peso ao final do teste (PEFI), na análise do GMD de suínos Landrace, Large White e Pietrain, submetidos a teste de granja.

\section{Material e Métodos}

Os dados de produção de suínos utilizados no presente trabalho pertencem à Granja Rezende S.A., que desenvolve atividades agropecuárias em Uberlândia (MG).

Foram utilizados para estudo registros de desempenho de 32.702 animais, sendo 17.836 da raça Landrace, nascidos entre agosto de 1991 e agosto de 1998, 9334 da raça Large White e 5532 da raça Pietrain, nascidos entre agosto de 1993 e agosto de 1998. Este rebanho foi formado a partir de importação de animais de ambos os sexos da Inglaterra (animais Landrace e Large White) e da Alemanha (animais Pietrain). A partir de 1993 o rebanho foi fechado.

Os animais avaliados nasceram em instalações climatizadas, sendo o procedimento de equalização de leitegada realizado em dois momentos (cinco horas e quatro dias após o nascimento). A desmama ocorreu com 14 dias de idade. Após a saída da creche (em torno de 64 dias de idade), os animais de cada sexo foram alojados, separadamente, em galpões de crescimento e terminação. Nestes galpões, o teste de granja foi iniciado com a pesagem dos animais após um período de adaptação de, aproximadamente, sete dias. Fêmeas e machos receberam ração à vontade em baias coletivas.

$\mathrm{Na}$ Tabela 1 pode ser observada a estrutura dos dados, após análises de consistência, para cada raça.

Foram realizadas análises de variância, utilizando-se o método de Quadrados Mínimos Ordinários, sendo considerada como variável dependente o GMD e, como variáveis independentes, o grupo contemporâneo (GC) e diferentes covariáveis (IDIN, IDFI, PEIN ou PEFI). O GC foi definido como animais de mesmo sexo, local, mês e ano de nascimento e grupo de teste. Estas análises, considerando cada covariável individualmente mais o GC, foram desenvolvidas com o objetivo de avaliar a importância de cada um dos efeitos sobre a variação do GMD.

Para estimação dos componentes de variância do GMD, os seguintes modelos estatísticos, cuja porção aleatória foi determinada em análises prévias (PITA, 2000), foram utilizados:

Para as raças Large White e Pietrain: $\mathrm{y}=\mathrm{X} \beta+$ $\mathrm{Z}_{1 \mathrm{~g}}+\mathrm{Wc}+\mathrm{e}$;

Para a raça Landrace: $\mathrm{y}=\mathrm{X} \beta+\mathrm{Z}_{1 \mathrm{~g}}+\mathrm{Z}_{2 \mathrm{~m}}+\mathrm{Wc}+\mathrm{e}$; em que: $\mathbf{y}$ é o vetor das observações; $\boldsymbol{\beta}$, o vetor dos efeitos fixos; $\mathbf{g}$, o vetor dos efeitos genéticos aditivos diretos dos animais; $\mathbf{m}$, o vetor dos efeitos genéticos maternos dos animais; $\mathbf{c}$, o vetor dos efeitos permanentes e comuns a membros da mesma leitegada; $\mathbf{e}$, o vetor dos efeitos residuais e; $\mathbf{X}, \mathbf{Z}_{\mathbf{1}}, \mathbf{Z}_{\mathbf{2}} \mathrm{e} \mathbf{W}$, matrizes de incidência que associam os efeitos apropriados a y.

As pressuposições para os modelos foram: $\mathrm{E}[\mathrm{y}]=\mathrm{X} \beta ; \mathrm{E}[\mathrm{g}]=0 ; \mathrm{E}[\mathrm{m}]=0 ; \mathrm{E}[\mathrm{c}]=0 ; \mathrm{E}[\mathrm{e}]=0$; $\operatorname{Var}[\mathrm{g}]=\mathrm{A} \sigma_{\mathrm{g}}^{2} ; \operatorname{Var}[\mathrm{m}]=\mathrm{A} \sigma^{2}{ }_{\mathrm{m}} ; \operatorname{Var}[\mathrm{c}]=\mathrm{INL} \sigma_{\mathrm{c}}^{2}$; $\operatorname{Var}[\mathrm{e}]=\mathrm{IN} \sigma_{\mathrm{e}}^{2} ; \operatorname{Cov}[\mathrm{g}, \mathrm{m}]=0$

em que: NL é número de leitegadas; $\boldsymbol{\sigma}_{\mathbf{g}} \mathbf{2}$, a variância genética aditiva direta; $\mathbf{N}$, o número de registros; $\sigma_{m}{ }^{2}$, a variância genética materna; $\mathbf{A}$, o numerador da matriz de parentesco; $\boldsymbol{\sigma}_{\mathbf{c}}{ }^{2}$, a variância de ambiente permanente e comum (leitegada); I, a matriz identidade; $\boldsymbol{\sigma}_{\mathrm{e}}^{2}$, a variância residual.

As covariâncias entre os efeitos genéticos e ambientais (permanentes e temporários), bem como entre os efeitos ambientais de diferentes animais, foram assumidas como zero.

$O$ vetor de efeitos fixos $(\beta)$ considerou a variável classificatória GC, mais cada uma das covariáveis individualmente. 
738 Rev. bras. zootec.

Tabela 1 - Estrutura dos dados analisados para cada raça

Table 1 - Analyzed data structure for each breed

\begin{tabular}{|c|c|c|c|c|c|c|}
\hline $\begin{array}{l}\text { Raça } \\
\text { Breed }\end{array}$ & $\begin{array}{l}\text { № de animais } \\
N \text {. of animals }\end{array}$ & $\begin{array}{l}\text { № de animais na } \mathrm{A}^{1} \\
N \text {. of animals in the } A^{1}\end{array}$ & $\begin{array}{l}\text { № de GC's } \\
N \text {. of } C G \text { 's }\end{array}$ & $\begin{array}{c}\text { № de leitegadas } \\
\text { N. of litters }\end{array}$ & $\begin{array}{l}\text { № de pais } \\
N \text {. of sires }\end{array}$ & $\begin{array}{c}\text { № de mães } \\
N \text {. of dams }\end{array}$ \\
\hline Landrace & 17.563 & 17.905 & 305 & 2708 & 154 & 1322 \\
\hline Large White & 8679 & 8894 & 213 & 1342 & 106 & 656 \\
\hline Pietrain & 5144 & 5317 & 190 & 1012 & 82 & 529 \\
\hline
\end{tabular}

${ }^{1} \mathrm{~A}$ - matriz de parentesco; ${ }^{2} \mathrm{GC}$ - grupo contemporâneo.

${ }^{1} A$ - relationship matrix; ${ }^{2} C G$ - contemporary group.

Os coeficientes de herdabilidade direto $\left(\mathrm{h}_{\mathrm{d}}{ }^{2}\right)$ e materno $\left(\mathrm{h}_{\mathrm{m}}{ }^{2}\right)$ e, o efeito de leitegada $\left(\mathrm{c}^{2}\right)$ foram calculados a partir das seguintes fórmulas:

$$
h_{d}^{2}=\frac{\sigma_{g}^{2}}{\sigma_{p}^{2}} \quad h_{m}^{2}=\frac{\sigma_{m}^{2}}{\sigma_{p}^{2}} \quad c^{2}=\frac{\sigma_{c}^{2}}{\sigma_{p}^{2}}
$$

em que $\sigma_{\mathbf{p}}{ }^{2}$ é variância fenotípica.

Os componentes de (co)variância foram estimados pelo método de Máxima Verossimilhança Restrita (REML) (PATTERSON e THOMPSON, 1971) não derivativo (SMITH e GRASER, 1986), aplicado a modelos animais, utilizando-se o programa computacional MTDFREML (BOLDMAN et al., 1993).

O programa computacional DFREML (MEYER, 1997) foi utilizado para obtenção dos erros-padrão aproximados das estimativas dos parâmetros genéticos e fenotípicos.

A escolha da covariável mais adequada para a análise genética do GMD foi realizada com base na comparação entre as análises unicaracterísticas para o GMD e, ainda, por meio de análises bicaracterísticas entre o GMD e o PEIN e o PEFI. Os modelos utilizados para análise destas duas últimas foram semelhantes aos utilizados para a análise do GMD em cada raça, mas considerando-se como covariáveis as características IDIN e IDFI, respectivamente, para PEIN e PEFI.

Componentes de variância não foram estimados para IDIN e IDFI, pois, nas condições do teste de granja realizado, estas características foram utilizadas como critério para a entrada e saída do teste dos animais. Portanto, não foi permitida a expressão destas pelos animais.

\section{Resultados e Discussão}

Na Tabela 2 podem ser observadas as médias e desvios-padrão para as características GMD, IDIN, IDFI, PEIN e PEFI, para cada raça. Pode-se observar que os animais Large White foram os que apresentaram maior GMD, seguidos dos Landrace e dos Pietrain. Os animais Landrace e Large White iniciaram e terminaram o teste com idades bem próximas (aproximadamente 70 e 144 dias, respectivamente), enquanto os animais Pietrain também iniciaram o teste com aproximadamente 70 dias, mas terminaram com aproximadamente 152 dias. Com relação às características PEIN e PEFI, os animais Landrace e Large White também apresentaram médias bastante próximas e os animais Pietrain, peso médio de, aproximadamente, 2,5 e 10,0 kg menor ao início e ao final do teste, respectivamente, quando comparados às outras duas raças.

Na Tabela 3 pode ser observado o resumo da análise de variância para a característica GMD, para as três raças.

Nas três raças verificou-se efeito significativo da variável classificatória GC, independentemente da covariável considerada.

Tabela 2 - Médias e desvios-padrão para ganho de peso médio diário (GMD), idade ao início (IDIN) e ao final (IDFI) do teste e peso ao início (PEIN) e ao final (PEFI) do teste, para cada raça

Table 2 - Averages and standard deviations for average daily gain (ADG), on-test age (ONA), off-test age (OFA), on-test weight (ONW), and off-test weight (OFW), for each breed

\begin{tabular}{lccccc}
\hline & \multicolumn{3}{c}{ Características } \\
& \multicolumn{3}{c}{ Traits } \\
\cline { 2 - 6 } Raça & GMD (kg/dia) & IDIN (dias) & IDFI (dias) & PEIN $(\mathrm{kg})$ & PEFI $(\mathrm{kg})$ \\
Breed & ODG (kg/day) & ONA (days) & OFA (days) & ONW $(\mathrm{kg})$ & OFW $(\mathrm{kg})$ \\
\hline Landrace & $0,866 \pm 0,103$ & $69,0 \pm 6,3$ & $144,8 \pm 4,9$ & $25,2 \pm 4,6$ & $90,7 \pm 10,5$ \\
Large White & $0,910 \pm 0,117$ & $70,8 \pm 6,4$ & $144,3 \pm 4,5$ & $25,0 \pm 4,9$ & $91,8 \pm 11,5$ \\
Pietrain & $0,713 \pm 0,089$ & $70,8 \pm 6,3$ & $152,0 \pm 5,1$ & $22,6 \pm 4,4$ & $80,4 \pm 9,2$ \\
\hline
\end{tabular}


PITA e ALBUQUERQUE

Tabela 3 - Fontes de variação e quadrados médios para o ganho de peso médio diário, nas raças Landrace, Large White e Pietrain

Table 3 - Sources of variation and mean squares for average daily gain, at Landrace, Large White, and Pietrain breeds

\begin{tabular}{|c|c|c|c|c|c|c|c|}
\hline \multirow{3}{*}{$\begin{array}{l}\operatorname{Raça}^{1} \\
\text { Breed }^{1}\end{array}$} & \multirow[b]{2}{*}{$\mathrm{GC}$} & \multicolumn{5}{|c|}{$\begin{array}{c}\text { Fontes de variação - Quadrados médios }{ }^{2} \\
\text { Sources of variation -Mean squares }{ }^{2}\end{array}$} & \multirow{3}{*}{$\begin{array}{l}\mathrm{R}^{2} \\
R^{2}\end{array}$} \\
\hline & & IDIN & IDFI & PEIN & PEFI & Resíduo & \\
\hline & $C G$ & ONA & $O F A$ & $O N W$ & $O F W$ & Residual & \\
\hline \multirow[t]{4}{*}{$\mathrm{LD}$} & $0,1486 * *$ & $0,0178^{\mathrm{NS}}$ & - & - & - & 0,0082 & 0,24 \\
\hline & $0,1360 * *$ & - & $0,0121^{\mathrm{NS}}$ & - & - & 0,0082 & 0,24 \\
\hline & $0,1421 * *$ & - & - & $8,6668 * *$ & - & 0,0077 & 0,29 \\
\hline & $0,1434 * *$ & - & - & - & $114,1571 * *$ & 0,0016 & 0,85 \\
\hline \multirow[t]{4}{*}{ LW } & $0,1350 * *$ & $0,4735 * *$ & - & - & - & 0,0106 & 0,24 \\
\hline & $0,1319 * *$ & - & $0,3316 * *$ & - & - & 0,0106 & 0,24 \\
\hline & $0,1251 * *$ & - & - & $8,9815 * *$ & - & 0,0096 & 0,31 \\
\hline & $0,1090 * *$ & - & - & - & $75,5573 * *$ & 0,0017 & 0,87 \\
\hline \multirow[t]{4}{*}{ PT } & $0,0454 * *$ & $0,0196^{\mathrm{NS}}$ & - & - & - & 0,0064 & 0,22 \\
\hline & $0,0389 * *$ & - & $0,0820 * *$ & - & - & 0,0063 & 0,22 \\
\hline & $0,0426 * *$ & - & - & $2,6834 * *$ & - & 0,0058 & 0,29 \\
\hline & $0,0461 * *$ & - & - & - & $25,7393 * *$ & 0,0012 & 0,85 \\
\hline
\end{tabular}

1 LD - Landrace, LW - Large White, PT - Pietrain.

2 GC - grupo contemporâneo, IDIN - idade ao início do teste, IDFI - idade ao final do teste.

PEIN - peso ao início do teste, PEFI - peso ao final do teste.

** significativo a $1 \%$, NS - não-significativo.

1 LD-Landrace, LW-Large White, PT-Pietrain.

2 CG- contemporary group, ONA- on-test age, OFA- off-test age, ONW-on-test weight. OFW- off-test weight.

** significant at $1 \%$ level, NS- non-significative.

Para a raça Landrace, as covariáveis IDIN e IDFI não responderam por porção significativa da variação do GMD, enquanto as covariáveis PEIN e PEFI influenciaram significativamente esta característica. Já para a raça Large White todas as covariáveis consideradas afetaram significativamente o GMD. $\mathrm{Na}$ raça Pietrain apenas a IDIN não respondeu por porção significativa da variação existente no GMD.

Para as três raças obteve-se, quando o modelo incluiu as variáveis independentes GC e PEFI, um coeficiente de determinação $\left(\mathrm{R}^{2}\right)$ alto e maior que os observados quando a IDIN, IDFI ou o PEIN foram utilizados como covariáveis.

Estes resultados sugerem que, embora a IDIN e a IDFI entre os animais seja bastante semelhante, pois é o critério adotado para entrada e saída do teste, estas respondem por porção importante da variação do GMD, à exceção da IDIN para as raças Landrace e Pietrain e da IDFI para a raça Landrace. Já o maior $\mathrm{R}^{2}$ observado, quando o PEFI foi considerado como covariável, pode indicar possível associação entre esta característica e o GMD.

Conforme pode ser observado nas Tabelas 4, 5 e 6 , respectivamente, para as raças Landrace, Large White e Pietrain, o efeito da inclusão no modelo das diferentes covariáveis sobre as estimativas dos parâmetros genéticos e fenotípicos para GMD foi bastante semelhante para as três raças. Assim, os dados apresentados nestas três Tabelas serão discutidos em conjunto.

Quando a covariável incluída no modelo de análise do GMD foi a IDIN ou a IDFI, as estimativas de herdabilidade do efeito direto $\left(\mathrm{h}_{\mathrm{d}}^{2}\right)$ e do efeito genético materno $\left(\mathrm{h}_{\mathrm{m}}{ }^{2}\right)$ (para a raça Landrace) e do efeito de ambiente permanente e comum $\left(\mathrm{c}^{2}\right)$ foram semelhantes às obtidas quando nenhuma covariável foi considerada no modelo.

Provavelmente esta semelhança nas estimativas é devida, como discutido anteriormente, ao fato de a IDIN e IDFI serem utilizadas como critério de entrada e saída dos animais no teste e, portanto, a semelhança entre os animais para essas características é bastante grande. Assim, embora os resultados da Tabela 3 tenham mostrado um efeito importante da IDIN e IDFI sobre o GMD da raça Large White, este não foi suficiente para afetar a estimação dos componentes de variância do GMD, quando estas características não foram consideradas como covariáveis.

Portanto, apesar de serem importantes fontes de variação do GMD, no caso particular destas três populações, a consideração ou não da IDIN ou IDFI, como covariáveis, é indiferente. 
Tabela 4 - Estimativas de variâncias, de parâmetros genéticos e fenotípicos e erros-padrão para ganho de peso médio diário, utilizando-se como covariáveis a idade ao início ou ao final do teste e o peso ao início ou ao final do teste, para a raça Landrace

Table 4 - Estimates of variances, genetic and phenotypic parameters, and standard errors for average daily gain, using as covariates the on-test age, off-test age, on-test weight, and off-test weight, for Landrace breed

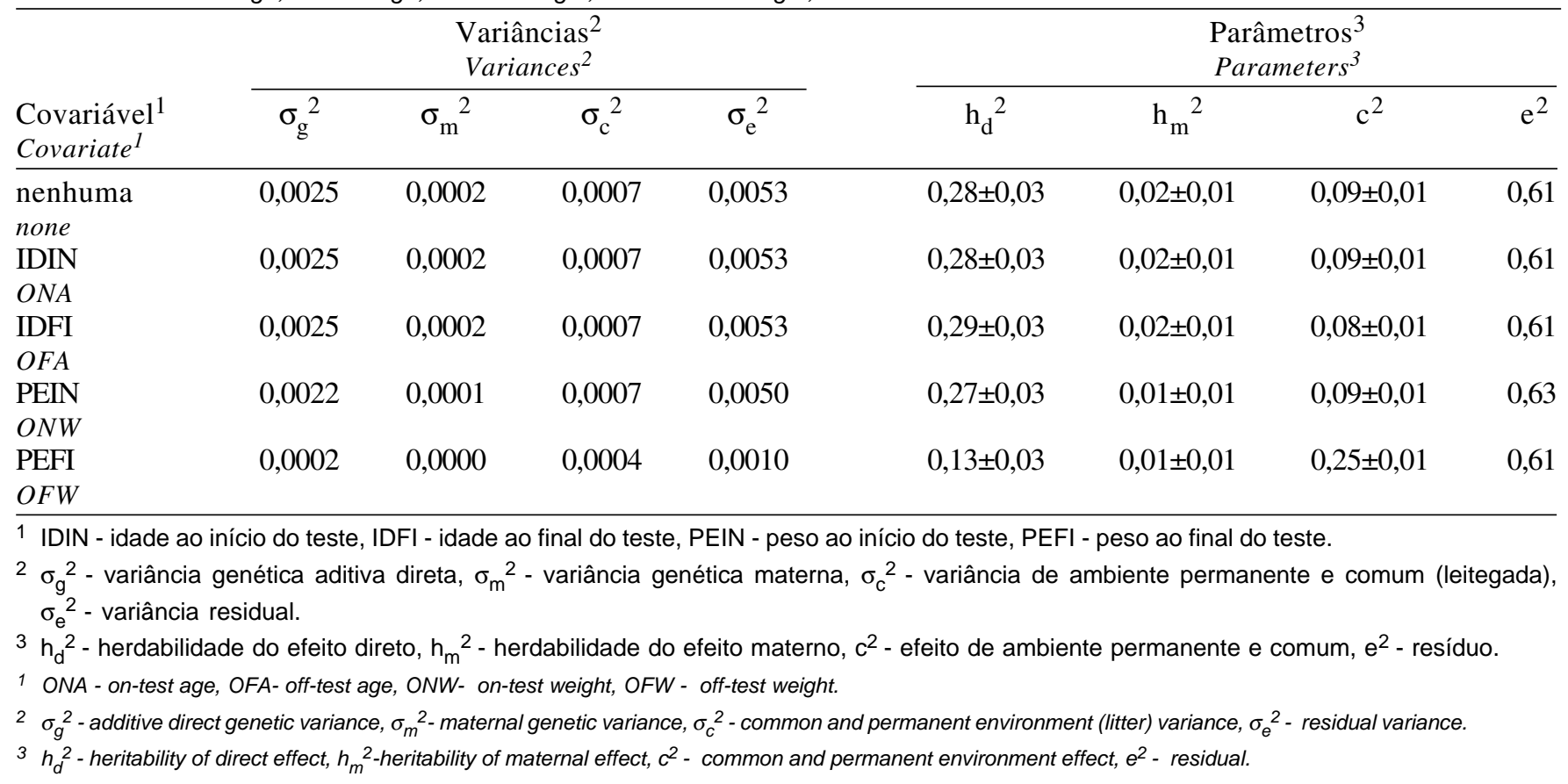

As estimativas de média magnitude observadas nesta pesquisa, para a herdabilidade do efeito direto utilizando-se estas covariáveis, foram semelhantes às verificadas por SILVA et al. (1992a), que consideraram a IDIN, como covariável, para análise do GMD de 4978 suínos Landrace, Large White e Duroc e, por JEFFRIES e PETTERSON (1982), ao analisarem informações de 2403 suínos Yorkshire e utilizarem a IDFI como covariável.

Já a inclusão do PEIN provocou redução nas variâncias genéticas aditiva direta e materna $\left(\sigma_{\mathrm{g}}{ }^{2} \mathrm{e}\right.$ $\sigma_{\mathrm{m}}{ }^{2}$, respectivamente) existentes nas populaçõ̃es, acarretando em pequena diminuição nas estimativas de $h_{d}{ }^{2}$ e $h_{m}{ }^{2}$. Entretanto, proporcionalmente e em relação ao modelo que incluiu como covariável a IDIN, verifica-se que esta redução na $\sigma_{\mathrm{g}}^{2}$ e $\sigma_{\mathrm{m}}{ }^{2}$ foi bastante considerável nas três raças (redução entre $12 \mathrm{e} 15 \%$ para $\sigma_{\mathrm{g}}{ }^{2}$ e de $50 \%$ para $\sigma_{\mathrm{m}}{ }^{2}$ ). Por outro lado, esta redução não foi tão pronunciada para a variância de ambiente permanente e comum (8,3\% para Large White e sem redução para Landrace e Pietrain) e residual (entre 5 e $6 \%$, aproximadamente).

As estimativas de $\mathrm{h}_{\mathrm{d}}{ }^{2}$ entre $0,26 \mathrm{e} 0,27$, observadas quando o PEIN foi considerado como covariável, foram semelhantes às verificadas por DUCOS et al. (1993), ao analisarem informações de GMD de suínos Landrace e
Large White também utilizando essa covariável, mas superiores à estimativa de 0,19 observada por BRAZÃO (1997), ao analisar o GMD de 2082 suínos Large White, utilizando o PEIN como covariável.

Quando o PEFI foi incluído como covariável no modelo, verificou-se redução na $\sigma_{\mathrm{g}}{ }^{2}$ e $\sigma_{\mathrm{m}}{ }^{2}$ bastante grande, provocando também diminuição expressiva nas estimativas de $\mathrm{h}_{\mathrm{d}}{ }^{2}$, em relação aos modelos em que a IDIN foi considerada como covariável. Entretanto, embora a variância de ambiente permanente e comum $\left(\sigma_{\mathrm{c}}{ }^{2}\right)$ também tenha sido reduzida, pode ser observado grande incremento do efeito de ambiente permanente e comum $\left(\mathrm{c}^{2}\right)$ (efeito de leitegada). Verificou-se também, diferentemente do esperado, redução muito pequena do resíduo $\left(\mathrm{e}^{2}\right)$, embora o decréscimo da variância residual $\left(\sigma_{\mathrm{e}}^{2}\right)$ tenha sido bastante expressivo. Ainda, a utilização do PEFI provocou redução bastante grande na variância total (fenotípica) do GMD nas três populações.

Nas três raças, a redução aproximada da $\sigma_{\mathrm{g}}{ }^{2}$, quando o PEFI foi considerado como covariável, foi entre 92 e $95 \%$ e da $\sigma_{\mathrm{m}}{ }^{2}$ para a raça Landrace, de $100 \%$. Já a redução da variância de leitegada foi entre 43 e $58 \%$ e da variância residual, em torno de $82 \%$.

Estimativas de $\mathrm{h}_{\mathrm{d}}{ }^{2}$ superiores às observadas nas Tabelas 4, 5 e 6, ao utilizar-se o PEFI como covariável, 
PITA e ALBUQUERQUE

Tabela 5 - Estimativas de variâncias de parâmetros genéticos e fenotípicos e erros-padrão para ganho de peso médio diário, utilizando-se como covariáveis a idade ao início ou ao final do teste e o peso ao início ou ao final do teste, para a raça Large White

Table 5 - Estimates of variances, genetic and phenotypic parameters, and standard errors for average daily gain, using as covariates the on-test age, off-test age, on-test weight, and off-test weight, for Large White breed

\begin{tabular}{|c|c|c|c|c|c|c|}
\hline \multirow[b]{2}{*}{$\begin{array}{l}\text { Covariável }^{1} \\
\text { Covariate }^{1}\end{array}$} & \multicolumn{3}{|c|}{$\begin{array}{l}\text { Variâncias } \\
\text { Variances }^{2}\end{array}$} & \multicolumn{3}{|c|}{$\begin{array}{l}\text { Parâmetros }{ }^{3} \\
\text { Parameters }^{3}\end{array}$} \\
\hline & $\sigma_{\mathrm{g}}^{2}$ & $\sigma_{\mathrm{c}}^{2}$ & $\sigma_{\mathrm{e}}^{2}$ & $\mathrm{~h}_{\mathrm{d}}{ }^{2}$ & $c^{2}$ & $\mathrm{e}^{2}$ \\
\hline $\begin{array}{l}\text { Nenhuma } \\
\text { none }\end{array}$ & 0,0032 & 0,0013 & 0,0069 & $0,28 \pm 0,04$ & $0,11 \pm 0,01$ & 0,61 \\
\hline $\begin{array}{l}\text { IDIN } \\
\text { ONA }\end{array}$ & 0,0032 & 0,0012 & 0,0069 & $0,28 \pm 0,04$ & $0,11 \pm 0,01$ & 0,61 \\
\hline $\begin{array}{l}\text { IDFI } \\
\text { OFA }\end{array}$ & 0,0032 & 0,0012 & 0,0069 & $0,28 \pm 0,04$ & $0,11 \pm 0,01$ & 0,61 \\
\hline $\begin{array}{l}\text { PEIN } \\
\text { ONW }\end{array}$ & 0,0028 & 0,0011 & 0,0065 & $0,27 \pm 0,04$ & $0,10 \pm 0,01$ & 0,63 \\
\hline $\begin{array}{l}\text { PEFI } \\
\text { OFW }\end{array}$ & 0,0002 & 0,0005 & 0,0012 & $0,13 \pm 0,03$ & $0,27 \pm 0,01$ & 0,60 \\
\hline
\end{tabular}

1 IDIN - idade ao início do teste, IDFI - idade ao final do teste, PEIN - peso ao início do teste, PEFI- peso ao final do teste.

${ }^{2} \sigma_{g}{ }^{2}$ - variância genética aditiva direta, $\sigma_{c}{ }^{2}$ - variância de ambiente permanente e comum (leitegada), $\sigma_{e}{ }^{2}$ - variância residual.

$3 \mathrm{~h}_{\mathrm{d}}{ }^{2}$ - herdabilidade do efeito direto, $\mathrm{c}^{2}$ - efeito de ambiente permanente e comum, $\mathrm{e}^{2}$ - resíduo.

1 ONA- on-test age, OFA- off-test age, ONW - on-test weight, OFW - off-test weight.

$2 \sigma_{g}{ }^{2}$ - additive direct genetic variance, $\sigma_{c}{ }^{2}$ - common and permanent environment (litter) variance, $\sigma_{e}{ }^{2}$ - residual variance.

${ }^{3} h_{d}^{2}$ - heritability of direct effect, $c^{2}$ - common and permanent environment effect, $e^{2}$ - residual.

Tabela 6 - Estimativas de variâncias de parâmetros genéticos e fenotípicos e erros-padrão para ganho de peso médio diário, utilizando-se como covariáveis a idade ao início ou ao final do teste e o peso ao início ou ao final do teste, para a raça Pietrain

Table 6 - Estimates of variances, genetic and phenotypic parameters, and standard errors for average daily gain, using as covariates the on-test age, off-test age, on-test weight, and off-test weight, for Pietrain breed

\begin{tabular}{|c|c|c|c|c|c|c|}
\hline \multirow[b]{2}{*}{$\begin{array}{l}\text { Covariável }^{1} \\
\text { Covariate }^{1}\end{array}$} & \multicolumn{3}{|c|}{$\begin{array}{l}\text { Variâncias }^{2} \\
\text { Variances }^{2}\end{array}$} & \multicolumn{3}{|c|}{$\begin{array}{l}\text { Parâmetros } \\
\text { Parameters }^{3}\end{array}$} \\
\hline & $\sigma_{g}^{2}$ & $\sigma_{\mathrm{c}}^{2}$ & $\sigma_{\mathrm{e}}^{2}$ & $\mathrm{~h}_{\mathrm{d}}^{2}$ & $c^{2}$ & $\mathrm{e}^{2}$ \\
\hline $\begin{array}{l}\text { Nenhuma } \\
\text { none }\end{array}$ & 0,0020 & 0,0010 & 0,0038 & $0,29 \pm 0,05$ & $0,15 \pm 0,02$ & 0,56 \\
\hline $\begin{array}{l}\text { IDIN } \\
\text { ONA }\end{array}$ & 0,0020 & 0,0010 & 0,0038 & $0,29 \pm 0,04$ & $0,15 \pm 0,02$ & 0,56 \\
\hline $\begin{array}{l}\text { IDFI } \\
O F A\end{array}$ & 0,0020 & 0,0010 & 0,0038 & $0,29 \pm 0,04$ & $0,15 \pm 0,02$ & 0,56 \\
\hline $\begin{array}{l}\text { PEIN } \\
O N W\end{array}$ & 0,0017 & 0,0010 & 0,0036 & $0,26 \pm 0,04$ & $0,16 \pm 0,02$ & 0,58 \\
\hline $\begin{array}{l}\text { PEFI } \\
O F W\end{array}$ & 0,0001 & 0,0005 & 0,0007 & $0,10 \pm 0,04$ & $0,38 \pm 0,02$ & 0,52 \\
\hline
\end{tabular}

1 IDIN - idade ao início do teste, IDFI - idade ao final do teste, PEIN - peso ao início do teste, PEFI - peso ao final do teste.

$2 \sigma_{g}{ }^{2}$ - variância genética aditiva direta, $\sigma_{c}^{2}$ - variância de ambiente permanente e comum (leitegada), $\sigma_{e}{ }^{2}-$ variância residual.

${ }^{3} \mathrm{~h}_{\mathrm{d}}{ }^{2}$ - herdabilidade do efeito direto, $\mathrm{c}^{2}$ - efeito de ambiente permanente e comum, $\mathrm{e}^{2}$ - resíduo.

1 ONA - on-test age, OFA- off-test age, ONW - on-test weight, OFW - off-test weight.

${ }^{2} \sigma_{g}{ }^{2}$ - additive direct genetic variance, $\sigma_{c}{ }^{2}$ - common and permanent environment (litter) variance, $\sigma_{e}{ }^{2}$ - residual variance.

${ }^{3} h_{d}^{2}$ - heritability of direct effect, $c^{2}$ - common and permanent environment effect, $e^{2}$ - residual. 
742 Rev. bras. zootec.

embora ainda de baixa magnitude, foram observadas por HOFER et al. (1992), que, utilizando amostras de uma população grande de suínos Yorkshire e Landrace, verificaram estimativas de 0,16 e 0,20 , respectivamente, ao analisarem o GMD considerando o PEFI como covariável.

Estes resultados sugerem que, assim como quando o PEIN foi utilizado como covariável, o uso do PEFI também implica em redução bem maior das variâncias genéticas, em relação às não genéticas.

Este comportamentoé bem diferente do esperado, ao se utilizar uma característica como covariável, já que o uso de covariáveis nos modelos de análise tem por objetivo diminuir a variância residual e, conseqüentemente, permitir melhor estimação dos efeitos genéticos envolvidos na característica sob análise.

Na Tabela 7 pode ser observado que tanto o PEIN quanto o PEFI apresentaram $\mathrm{h}_{\mathrm{d}}{ }^{2}$ de média magnitude (à exceção do PEIN para a raça Landrace, cuja herdabilidade foi baixa), sendo estas próximas às observadas para o GMD, quando a IDIN foi utilizada como covariável (Tabelas 4, 5 e 6). Estes resultados sugerem existir considerável variação genética e bastante semelhante para essas três características nas populações estudadas (principalmente para GMD e PEFI).
As correlações genéticas $\left(r_{g}\right)$ e fenotípicas $\left(r_{p}\right)$ observadas entre o GMD e o PEIN foram de, respectivamente, 0,46 e 0,26 para os animais Landrace (LD), 0,40 e 0,30 para os animais Large White (LW) e, 0,51 e 0,30 para os animais Pietrain (PT). Já entre o GMD e o PEFI, estas foram de 0,96 e 0,91 para LD, 0,96 e 0,93 para LW e 0,97 e 0,92 para PT. As correlações próximas de 1,0 entre GMD e PEFI, para as três raças, se explicam pelo fato de o GMD ser um componente do PEFI.

A análise destes resultados sugere que as altas $r_{g}$ entre GMD e PEFI, para as três raças, justificam a grande redução na variância genética aditiva direta $\left(\sigma_{\mathrm{g}}^{2}\right)$ e, conseqüentemente, nas estimativas de $\mathrm{h}_{\mathrm{d}}^{2}$ do GMD, quando o PEFI foi incluído como covariável nos modelos de análise. Por outro lado, o efeito da inclusão do PEIN, como covariável nos modelos, sobre $\mathrm{h}_{\mathrm{d}}{ }^{2}$ do GMD foi muito pequena, embora as $r_{g}$ entre GMD e PEIN não tenham sido desprezíveis para as três raças.

Portanto, a utilização da IDIN ou IDFI como covariáveis permitiria a comparação dos animais quanto ao GMD em igualdade de condições (mesma idade fisiológica), mas sem afetar sua análise genética. Cabe ainda ressaltar que, apesar de aparentemente a utilização da IDFI como covariável ser um

Tabela 7 - Estimativas de variâncias e de parâmetros genéticos e fenotípicos para peso ao início e ao final do teste, para as raças Landrace, Large White e Pietrain

Table 7 - Estimates of variances and genetic and phenotypic parameters for on-test weight, and off-test weight, for Landrace, Large White, and Pietrain breeds

\begin{tabular}{|c|c|c|c|c|c|c|c|c|c|}
\hline \multirow[b]{2}{*}{$\begin{array}{l}\text { Raça } \\
\text { Breed }^{1}\end{array}$} & \multirow[b]{2}{*}{$\begin{array}{c}\text { Característica }^{2} \\
\text { Trait }^{2}\end{array}$} & \multicolumn{4}{|c|}{$\begin{array}{c}\text { Variâncias }^{2} \\
\text { Variances }^{2}\end{array}$} & \multicolumn{4}{|c|}{$\begin{array}{l}\text { Parâmetros }^{3} \\
\text { Parameters }^{3}\end{array}$} \\
\hline & & $\sigma_{\mathrm{g}}^{2}$ & $\sigma_{\mathrm{m}}^{2}$ & $\sigma_{\mathrm{c}}^{2}$ & $\sigma_{\mathrm{e}}^{2}$ & $\mathrm{~h}_{\mathrm{d}}^{2}$ & $\mathrm{~h}_{\mathrm{m}}{ }^{2}$ & $c^{2}$ & $\mathrm{e}^{2}$ \\
\hline \multirow[t]{3}{*}{$\overline{\mathrm{LD}}$} & PEIN & & & & & & & & \\
\hline & $\begin{array}{l}O N W \\
\text { PEFI }\end{array}$ & 1,388 & 0,774 & 1,920 & 8,320 & 0,11 & 0,06 & 0,16 & 0,67 \\
\hline & $O F W$ & 21,161 & 2,650 & 6,917 & 45,382 & 0,28 & 0,03 & 0,09 & 0,60 \\
\hline \multirow[t]{3}{*}{ LW } & PEIN & & & & & & & & \\
\hline & $\begin{array}{l}\text { ONW } \\
\text { PEFI }\end{array}$ & 2,831 & - & 2,724 & 8,385 & 0,20 & - & 0,20 & 0,60 \\
\hline & $O F W$ & 26,872 & - & 12,920 & 54,027 & 0,29 & - & 0,14 & 0,57 \\
\hline \multirow[t]{3}{*}{ PT } & PEIN & & & & & & & & \\
\hline & $\begin{array}{l}O N W \\
\text { PEFI }\end{array}$ & 1,881 & - & 2,733 & 6,126 & 0,18 & - & 0,25 & 0,57 \\
\hline & $O F W$ & 19,386 & - & 11,414 & 37,341 & 0,28 & - & 0,17 & 0,55 \\
\hline
\end{tabular}

\footnotetext{
${ }_{1}^{1}$ LD - Landrace, LW - Large White, PT - Pietrain.
}

2 PEIN - peso ao início do teste, PEFI - peso ao final do teste.

$3 \sigma_{\mathrm{g}}^{2}$ - variância genética aditiva direta, $\sigma_{\mathrm{m}}{ }^{2}$ - variância genética materna, $\sigma_{\mathrm{c}}{ }^{2}$ - variância de ambiente permanente e comum, $\sigma_{\mathrm{e}}{ }^{2}$ - variância residual.

${ }_{4} \mathrm{~h}_{\mathrm{g}}{ }^{2}$ - herdabilidade do efeito direto, $\mathrm{h}_{\mathrm{m}}$ - herdabilidade do efeito materno, $\mathrm{c}^{2}$ - efeito de ambiente permanente e comum, $\mathrm{e}^{2}-$ resíduo.

1 LD - Landrace, LW - Large White, $P T$ - Pietrain.

2 ONW - on-test weight, OFW - off-test weight.

${ }_{3}^{3} \sigma_{g}^{2}$ - additive direct genetic variance, $\sigma_{m}{ }^{2}$ - maternal genetic variance, $\sigma_{c}{ }^{2}$ - common and permanent environment (litter) variance, $\sigma_{e}{ }^{2}$ - residual variance.

${ }^{4} h_{d}^{2}$ - heritability of direct effect, $h_{m}{ }^{2}$ - heritability of maternal effect, $c^{2}$ - common and permanent environment effect, $e^{2}$ - residual. 
contra-senso, já que esta é uma medida realizada após a expressão do GMD pelos animais, esta é semelhante, neste caso, à IDIN, pois, nas condições do teste de granja em que os animais foram avaliados, o período de teste é pouco variável.

Já a utilização do PEIN, apesar de reduzir o efeito do ganho compensatório que alguns animais poderiam apresentar, afetaria também o resultado da avaliação genética dos animais geneticamente superiores para GMD, penalizando-os.

\section{Conclusões}

As características idade ao início ou ao final do teste, quando consideradas como covariáveis para análise do GMD de suínos submetidos a teste de granja, permitem o estudo desta característica sem que sua variabilidade genética seja afetada. Diferentemente, tanto o peso ao início quanto ao final do teste provocam maior redução na variabilidade genética do GMD, comparativamente à observada para a variância residual, afetando assim as análises genéticas desta característica.

\section{Agradecimento}

À FAPESP (Fundação de Amparo à Pesquisa do Estado de São Paulo), pelo financiamento desta pesquisa, e à Granja Rezende S.A., pelo fornecimento dos dados utilizados neste estudo.

\section{Referências Bibliográficas}

BIDANEL, J.P., DUCOS, A. 1996. Genetic correlations between test station and on-farm performance traits in Large White and French Landrace pig breeds. Livest. Prod. Sci., 45(1):55-62.

BOLDMAN, K.G., KRIESE, L.A., VAN VLECK, L.D. et al. 1993. A manual for use of MTDFREML. Lincoln: USDAARS. $120 \mathrm{p}$.

BRANDT, H., TÄUBERT, H. 1998. Parameter estimates for purebred and crossbred performances in pigs. J. Anim. Breed. Gen., 115(2):97-104.

BRAZÃO, C.S. Estimativas de parâmetros genéticos e ambientais para algumas características de desempenho em suínos da raça Large White. Jaboticabal, SP: FCAV, 1997.68p. Dissertação (Mestrado em Zootecnia) - Faculdade de Ciências Agrárias e Veterinárias/Universidade Estadual Paulista, 1997.

CAMERON, N.D. 1990. Comparison of Duroc and British Landrace pigs and the estimation of genetic and phenotypic parameters for growth and carcass traits. Anim. Prod., 50(1):141-153.

COSTA, C.N., FÁ VERO, J.A., LARRAMBEBERE, W.H.S. et al. 1986. Evolução das características de desempenho medidas nos testes de reprodutores suínos em Santa Catarina. Pesq. Agrop. Bras., 21(1):79-85.
DUCOS, A., BIDANEL, J.P., DUCROCQ, V. et al. 1993 Multivariate restricted maximum likelihood estimation of genetic parameters for growth, carcass and meat quality traits in French Large White and French Landrace pigs. Gen. Sel. Evol., 25:475-493.

GU, Y., HALEY, C.S., THOMPSON, R. 1989. Estimates of genetic and phenotypic parameters of growth and carcass traits from closed lines of pigs on restricted feeding. Anim. Prod., 49(3):467-475.

GUERRA, D., DIEGUEZ, F.J., SANTANA, I. et al. 1992 Genetic and phenotypic parameters of a pig paternal synthetic breed. Cuban J. Agric. Sci., 26(1):11-15.

HOFER, A., HAGGER, C., KÜNZI, N. 1992. Genetic evaluation of on-farm tested pigs using an animal model. I. Estimation of variance components with restricted maximum likelihood. Livest. Prod. Sci., 30(1/2):69-82.

JEFFRIES, D.C, PETERSON, R.G. 1982. Heritabilities and genetic correlations for ultrasonic backfat measurements, growth and carcass traits in swine. Canadian J. Anim. Sci., 62(3):665-670.

LO, L.L., MCLAREN, D.G., MCKEITH, F.K. et al. 1992. Genetic analyses of growth, real-time ultrasound, carcass, and pork quality traits in Duroc and Landrace pigs: II- Heritabilities and correlations. J. Anim. Sci., 70(8):2387-2396.

MERKS, J.W.M. 1987. Genotype X environment interactions in pig breeding programmes. II. Environmental effects and genetic parameters in central test. Livet. Prod.Sci., 16(3):215-228.

MEYER, K. DFREML: Usernotes. 1997.29p.

PATTERSON, H.D., THOMPSON, R. 1971. Recovery of inter-block information when block sizes are unequal. Biometrika, 58:545-554.

PITA, F.V.C. Modelos para avaliação genética e comparação de características de desempenho para a seleção de suínos. Jaboticabal, SP: FCAV, 2000. 157p. Dissertação (Mestrado em Zootecnia) - Faculdade de Ciências Agrárias e Veterinárias/Universidade Estadual Paulista, 2000.

ROSO, V.M. Parâmetros genéticos para ganho de peso médio diário e espessura de toucinho em suínos de diferentes grupos raciais. In: REUNIÃO ANUAL DA SOCIEDADEBRASILEIRADEZOOTECNIA, 34, 1997a, Juiz de Fora. Anais...Juiz de Fora: SBZ, 1997. p.332-334.

ROSO, V.M. Alternativas para avaliar taxa de crescimento e conseqüências na seleção de suínos. In: REUNIÃO ANUAL DA SOCIEDADE BRASILEIRA DE ZOOTECNIA, 34, 1997b, Juiz de Fora. Anais...Juiz de Fora: SBZ, 1997.p.308-310.

SILVA, M.A., CATALAN, G., TORRES, R.A. et al. 1992a Estimativas de componentes genéticos de características de importância econômica, em três diferentes raças de suínos. R. Soc. Bras. Zootec., 21(5):923-932.

SILVA, M.A., CATALAN, G., TORRES, R.A.et al. 1992b. Fatores que influem no desempenho de suínos das raças Landrace, Large White e Duroc. I- Características produtivas. R. Soc. Bras. Zootec., 21(5):912-922.

SMITH, S.P., GRASER, H.U. 1986. Estimating variance components in a class of mixed models by restricted maximum likelihood. J. Dairy Sci., 69(4):1156-1165.

Recebido em: 28/03/00

Aceito em: 21/12/00 